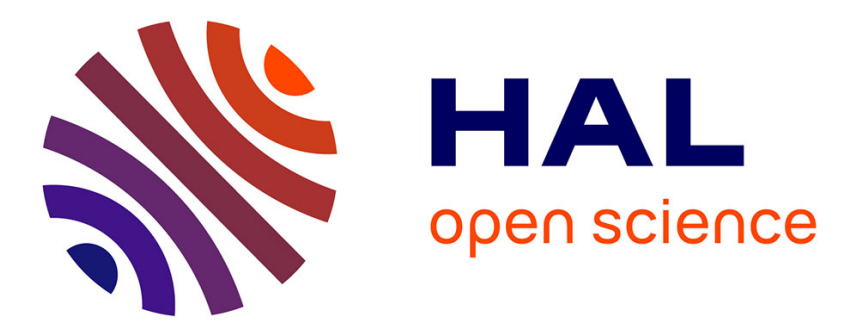

\title{
Fragmentation cross sections of protonated water clusters
}

\author{
Sébastien Zamith, Pierre Labastie, Jean-Marc L'Hermite
}

\section{To cite this version:}

Sébastien Zamith, Pierre Labastie, Jean-Marc L'Hermite. Fragmentation cross sections of protonated water clusters. Journal of Chemical Physics, 2012, 136 (21), pp.214301. 10.1063/1.4722886 . hal00703409

\section{HAL Id: hal-00703409 \\ https://hal.science/hal-00703409}

Submitted on 1 Jun 2012

HAL is a multi-disciplinary open access archive for the deposit and dissemination of scientific research documents, whether they are published or not. The documents may come from teaching and research institutions in France or abroad, or from public or private research centers.
L'archive ouverte pluridisciplinaire HAL, est destinée au dépôt et à la diffusion de documents scientifiques de niveau recherche, publiés ou non, émanant des établissements d'enseignement et de recherche français ou étrangers, des laboratoires publics ou privés. 


\title{
Fragmentation cross sections of protonated water clusters
}

\author{
Sébastien Zamith ${ }^{1,2}$, Pierre Labastie ${ }^{1,2}$, and Jean-Marc L'Hermite ${ }^{1,2}$ \\ ${ }^{1}$ Université de Toulouse ; UPS ; Laboratoire Collisions Agrégats Réactivité, IRSAMC ; F-31062 \\ Toulouse, France \\ ${ }^{2}$ CNRS ; UMR 5589 ; F-31062 Toulouse, France
}

\begin{abstract}
We have measured fragmentation cross-sections of protonated water cluster cations $\left(\mathrm{H}_{2} \mathrm{O}\right)_{n=30-50} \mathrm{H}^{+}$by collision with water molecules. The clusters have well defined sizes and internal energies. The collision energy has been varied from 0.5 to $300 \mathrm{eV}$. We also performed the same measurements on deuterated water clusters $\left(\mathrm{D}_{2} \mathrm{O}\right)_{n=5-45} \mathrm{D}^{+}$colliding with deuterated water molecules. The main fragmentation channel is shown to be a sequential thermal evaporation of single molecules following an initial transfer of relative kinetic energy into internal energy of the cluster. Unexpectedly, that initial transfer is very low on average, of the order of $1 \%$ of collision energy. We evaluate that for direct collisions (i.e. within the hard sphere radius), the probability for observing no fragmentation at all is more than $35 \%$, independently of cluster size and collision energy, over our range of study. Such an effect is well known at higher energies, where it is attributed to electronic effects, but has been reported only in a theoretical study of the collision of helium atoms with sodium clusters in that energy range, where only vibrational excitation occurs.
\end{abstract}

\section{Introduction}

When a molecule, an atom or an ion collides with a solid target, it loses its energy in small steps through collisions with the constituents of the target. At energies above $1 \mathrm{keV}$ or so, the energy loss is mainly through electronic excitation of the target. The energy loss per unit length (the "stopping power") increases as the velocity decreases and reaches a maximum, which leads to the well-known "Bragg peak" at some penetration depth. At this point, the particle loses so much energy that it may damage or otherwise modify the target. Afterwards, the projectile keeps flying, with much less energy, in the $10-100 \mathrm{eV}$ range. Electronic excitation is then impossible, so that the energy loss is through "nuclear" (or "vibrational") excitation of the target. This type of excitation does not lead to mere structural modification of the target, only to some heating, which is almost not observable in bulk targets.

Collisions between clusters and molecules, atoms or ions lead basically to the same effects. High energy collisions, where electronic excitation occurs, have been widely studied, both experimentally and theoretically $[1,2,3,4,5,6,7$ and references therein]. In those studies, an effect specific to binary collisions, charge exchange, often occurs $[1,8]$. Collisions in the energy range where nuclear excitation occurs have been much less studied per se [3,9]. On the other hand, they are often used as a mean to determine the stability of complexes, as in Ref.10. A variety of effects are expected. The impinging particle may:

(i) Kick out a constituent of the cluster: this is the mechanism of "direct" or "impulsive" fragmentation. A variant is a "direct exchange" between the projectile and one constituent of the cluster. 
(ii) Transfer some energy to the cluster, which is therefore heated, thus leading to the subsequent evaporation of one or more particles: this is the mechanism of "thermal fragmentation".

(iii) Attach to the cluster for some time. If this time is long enough to observe the resulting cluster, the collision is considered "sticking" or attaching. If the impinging molecule evaporates before the cluster is observed, some energy has nevertheless been transferred to the cluster and the subsequent behavior is the same as in case (ii). Another eventuality after attachment is that another constituent of the cluster is evaporated first, leading to "thermal exchange".

(iv) Undergo an almost elastic collision with the cluster, which leads to unobservable consequences in our experiment (no change of the mass of the cluster).

Since in all cases except (iv), the initial process may be followed by other evaporations, the different cases can only be discriminated experimentally if one is able to detect all the collision products in coincidence. Some of the studies performed at higher energies use coincidence devices [6], but all the works we are aware of in the low energy range are based on the observation of fragmentation patterns, as in Ref. 9, 10 and 11.

In this paper, we present the measurement of absolute fragmentation cross sections of positively charged protonated water clusters induced by collisions with water molecules. The clusters have a controlled internal energy, a well-defined size, and the collision energy in the center-of-mass (CM) frame can be accurately varied from $\sim 0.1 \mathrm{eV}$ to $300 \mathrm{eV}$. Fragmentation of water clusters induced by collisions with different targets has already been reported $[7,12,13,14]$. Dissociation cross-sections have been reported by Armentrout's group [15,16], for small (less than seven water molecules) protonated water clusters. Those experiments allow reaching very low collision energies, but lack control of the cluster temperature. In this size range, our cross section measurements are very close to theirs. We are able to reach much higher sizes, and to fix the clusters temperature, which allows a better control of the collision parameters.

The main result of our work is that in a wide collision energy range (roughly between $5 \mathrm{eV}$ and a few hundreds of $\mathrm{eV}$ ), and for roughly one third of the head-on (i.e. inside the geometrical cross section of the cluster) collisions, such a small amount of the collision energy is transferred into internal energy of the cluster, that the cluster does not fragment at all, i.e. case (iv) above is observed. The high rate of almost elastic collisions is very puzzling, since simple models of clustermolecule collisions lead to a significant energy transfer for head-on collisions. One of those models, the line-of-centers one [17] is used below to model the shape of the cross section as a function of energy.

An amazing "transparency effect" has been predicted in Ref. 3 for sodium cluster, where the projectile goes all the way through the target almost without loss of energy. While the electronic and spatial structures are very different in sodium and in water, it is possible that we observe a similar process. Actually, this effect has been reported in Ref. 6 in the case of fullerene- $-\mathrm{He}^{+}$collisions at high energy, although the observation is based on the measurement of relative cross-sections, while absolute measurements would be more conclusive. In Ref. 7, the authors have studied the collision of protonated water clusters at high collision energy with $\mathrm{He}$ and $\mathrm{Xe}$ atoms and observed a transparency effect. Absolute cross sections have been measured in Ref. 9 for fullerene-rare gas collisions, and are again much lower than the geometric cross section. It should be mentioned that channeling effects [18], i.e. the almost lossless travel of an ion through a macroscopic crystal, have long been known. Those effects are very similar to Saalmann and Schmidt's [3] transparency effect. 


\section{Experimental setup}

The experimental setup has already been detailed elsewhere [19]. Water clusters are produced in a gas aggregation source. Right after production, they enter a cell whose temperature can be varied between 25 and $400 \mathrm{~K}$. In this cell, clusters undergo thousands of collisions with the carrier gas, which is enough to accurately fix their temperature. The clusters are then mass-selected and their velocity can be reduced, using a special electrostatic device [19], to reach kinetic energies as low as a few tenths of an $\mathrm{eV}$ in the $\mathrm{CM}$ frame. At this point, we get clusters with a known size, a known kinetic energy and a Boltzmann internal energy distribution at a known temperature. Those clusters then enter a $5 \mathrm{~cm}$ long collision cell that contains a controlled pressure of water vapor. Typical values for the water vapor pressure in the cell range from 1 to $3.10^{-4} \mathrm{mbar}$ in order to keep the average number of collisions below unity. In the case of water, evaporation occurs before clusters reach the collision cell when their temperature is above roughly $130 \mathrm{~K}$; to prevent this phenomenon, clusters' temperature has been kept below $130 \mathrm{~K}$ all along the experiments. These clusters undergo collisions with the water molecules of the vapor, and the products are mass analyzed in a high resolution time of flight mass spectrometer. From these mass spectra, absolute cross sections of the reaction that took place in the cell are deduced. Two kinds of reactions can be studied. First, owing to the very low collision energy, long lived products can be created, which allows measuring attachment cross sections $[20,21,22]$. Second, fragmentation cross sections can also be measured at higher collision energies, whose analysis is the aim of this paper.

\section{Computation and calibration of fragmentation cross sections}

Our raw experimental data are fragmentation patterns such as that of $\left(\mathrm{H}_{2} \mathrm{O}\right)_{30} \mathrm{H}^{+}$displayed in figure 1 for different $\mathrm{CM}$ collision energies. To deduce absolute cross-sections, we use a procedure close to that of Kinsey et al [23]. We recall it briefly: first, for a given incident cluster's velocity $v_{0}$, an apparent fragmentation cross section is deduced from the mass spectra by the relation:

$$
\sigma_{a p p}\left(v_{0}\right)=-\frac{\ln \left(I / I_{0}\right)}{\rho l}
$$

where $I / I_{0}$ is the ratio between the integrated intensity of parent $I$ and the total integrated intensity $I_{0}$, including the fragments and the parent, $\rho$ is the density of the vapor in the collision cell, $l$ is the length of this cell.

Relation (1) must then be corrected in order to account for two factors: a) the velocity distribution of molecular targets, and $b$ ) the velocity dependence of cross sections.

a) The velocity $v_{0}$ is associated to a mean relative velocity:

$$
\bar{v}=v_{0}\left[\operatorname{erf}(\sqrt{a})+\frac{1}{2 a} \operatorname{erf}(\sqrt{a})+\frac{e^{-a}}{\sqrt{\pi a}}\right]
$$

with $a=\frac{E_{l a b}}{n k_{B} T_{\text {cell }}}$, where $E_{l a b}$ is the kinetic energy of the cluster in the laboratory frame, $n$ is the size of the cluster (rigorously, it should be the ratio of the mass of the cluster to the mass of the molecule), $k_{B}$ is the Boltzmann constant and $T_{\text {cell }}$ is the temperature of the vapor in the cell, which is roughly $300 \mathrm{~K}$ in our case. 
b) To account for the velocity dependence of cross-sections, the "Kinsey factor" $f\left(v_{0}, T_{\text {cell }}\right)$ is introduced [23]. This factor, which depends on the shape of the function $\sigma(v)$, is very close to 1 , whenever $v_{0}$ is higher than the mean thermal velocity of the targets.

Finally, the cross-section at mean velocity $\bar{v}$ is deduced from the following relation [23]:

$$
v_{0} \sigma_{\text {app }}\left(v_{0}\right)=f\left(v_{0}, T_{\text {cell }}\right) \bar{v} \sigma(\bar{v})
$$

As soon as $\boldsymbol{a}>\mathbf{2 0}$, which corresponds to an incident energy of $15 \mathrm{eV}$ for a cluster of size 30 , all the correction factors are very close to 1 and can be dismissed. This criterion is valid provided that the cross-section is measured for collision energies high enough above the threshold for dissociation, which is the case in our experiment.

To apply Eq. (1), an important parameter must be determined, namely the product of the density $\rho$ and the length I. A careful determination of both parameters has been described in Ref. 20. We use the same calibration here. In particular we have checked that the quantity $-\ln \left(I / I_{0}\right)$ evolves linearly with $\rho l$ for a given collision energy and deduced the cross-section from the slope. An example of such a curve is given in figure 2 , for $n=30$ protonated clusters colliding with water molecules at a collision energy of $2.3 \mathrm{eV}$. From the slope of this curve the fragmentation crosssection is readily deduced as $125 \AA^{2}$. Since the cross-section is deduced from the slope of such a curve, there is no need in knowing the absolute density in the cell but rather to have a correct measurement of its variation. The density $\rho$ in the cell is deduced from the relation $\rho=P_{c e l} / k_{B} T_{c e l}$ where $P_{c e l}$ is the pressure measured in the collision cell, $T_{c e l}$ its temperature and $k_{B}$ the Boltzmann constant. The pressure is simultaneously measured by an ionization gauge (Leybold lonivac ITR 90) and a capacitive gauge (Leybold Ceravac CTR 91). The capacitive gauge provides accurate gas independent pressure variations. Of course the pressures given by these gauges might be off by an unknown amount, however, this would not affect the major conclusions of the present paper since fragmentation cross-sections can be directly compared with attachment cross-sections previously measured with exactly the same experimental setup and using the same data processing method. In particular the same gauges were used, so that any systematic error in pressure measurements would have the same effect in both experiments. Since all the attachment cross sections measured in Ref. 20 converge towards the same values at large sizes (see Fig. 9 of [20]), this common value is likely to be close to the geometrical cross section, which is calculated as follows: define an effective interaction radius $R^{*}=R_{c}+R_{0}$, where $R_{0}$ is the molecular radius and $R_{c}=R_{0} n^{1 / 3}$ is the cluster radius, assuming that the clusters are spherical; the geometrical cross section is then:

$$
\sigma_{g e o}=\pi R *^{2}
$$

In bulk ice, the value of $R_{0}$ is $1.98 \AA$. In Ref. 20, a slightly higher value has been found, namely $2.17 \AA$ for clusters in the $n \approx 100$ size range. However, it is shown in Ref. 20 (see Fig. 11 of this reference) that some models could lead to a higher cross section than geometrical. We therefore adopt a conservative attitude in this paper and use a geometrical cross section given by Eq. (4) and a value $R_{0}=1.98 \AA$. Doing this, the ratio of the measured cross section to the geometrical cross section might be overestimated by at most $17 \%$. This means that all the experimental ratios presented below are upper bounds for the actual ratios. Our point is that those experimental ratios are too low 
by about $35 \%$. Actual ratios might be even lower. Anyway, the absolute values deduced for the cross-sections compare well to values found in the literature (see section IV 1.).

One may be concerned by the influence of multiple collisions on the determination of the crosssections. Indeed, if for some collisions the energy transfer is rather inefficient, only a small portion of the collision energy is converted into internal energy of the clusters, which is just not enough to lead to fragmentation. But a second collision, still with a rather inefficient energy transfer, could induce fragmentation. Thus, multiple collisions might well artificially increase the measured cross-section. One way to ensure that one measures cross-sections in single collision conditions is to extrapolate the cross-sections measured at different pressures to zero pressure value. Equivalently, as already mentioned, we have checked that the quantity $-\ln \left(I / I_{0}\right)$ evolves linearly with the density in the collision cell (see Fig. 2). In any event, multiple collisions will induce a measured cross-section higher than it is actually.

Another issue with the way we compute $I_{0}$ is that we suppose that all the charged fragments are detected, so that the sum of all the detected intensities is the same as the intensity of the parent cluster entering the cell. This might not be so if either some fragment gets undetected because it has been deflected away, or if a small charged fragment is ejected, which cannot be detected. Another way to measure $I_{0}$, which is more experimentally demanding, is to take the intensity of the parent cluster when there is no gas in the cell, and compare it with the intensity $I$ at a given pressure in the cell. For this to be done, a reference mass spectrum at zero pressure must be recorded before and after each fragmentation spectrum. The results obtained are the same as previously, which indicates first that our measurements using Eq. (1) are valid, and second that the main evaporation channel is, unsurprisingly, the loss of small neutral monomers.

\section{Experimental Results}

\section{Fragmentation cross section}

The fragmentation cross sections have been measured for both protonated water clusters $\left(\mathrm{H}_{2} \mathrm{O}\right)_{n} \mathrm{H}^{+}$impinging on $\mathrm{H}_{2} \mathrm{O}$ water molecules and deuterated heavy water clusters $\left(\mathrm{D}_{2} \mathrm{O}\right)_{n} \mathrm{D}^{+}$impinging on $\mathrm{D}_{2} \mathrm{O}$ heavy water molecules. There is no major qualitative difference between both cases. The ratios of the fragmentation cross sections to the geometric cross sections $\sigma_{\text {frag }} / \sigma_{\text {geo }}$ for water clusters and deuterated water clusters are displayed in figure 3.

First of all let us compare our results with some available data from the literature. In ref. 15, Armentrout and coworkers report a fragmentation cross-section of $49 \AA^{2}$ for the collision of $\left(\mathrm{H}_{2} \mathrm{O}\right)_{5} \mathrm{H}^{+}$ with Xenon atoms at $20 \mathrm{eV}$ collision energy. In the present study, we find a cross-section of $55 \AA^{2}$ for $\left(D_{2} O\right)_{5} D^{+}$clusters colliding with $D_{2} \mathrm{O}$ molecules at $100 \mathrm{eV}$ collision energy. In [10] the authors give a value of about $70 \AA^{2}$ for the collision of $\mathrm{Cd}^{2+}\left(\mathrm{H}_{2} \mathrm{O}\right)_{10}$ in collisions with Xenon between 1 and $6 \mathrm{eV}$. This value compares rather well with the present results on $\left(\mathrm{D}_{2} \mathrm{O}\right)_{10} \mathrm{D}^{+}$since we measure a cross-section of $70 \AA$ as well. Of course we are aware that the comparisons are not strictly correct (different targets, $H / D$ difference). Nevertheless we believe that the agreement with previously measured crosssections tends to validate the present measurements.

The most noticeable feature in the evolution with collision energy of the fragmentation of water clusters is that, even at collision energies of hundreds of eV, the fragmentation cross section never reaches the geometrical cross section. Up to about $5 \mathrm{eV}$, the fragmentation unsurprisingly increases with collision energy, although the large size dependence of the curves is hard to interpret. For higher energies, the ratio $\sigma_{\text {frag }} / \sigma_{\text {geo }}$ reaches a plateau between roughly 10 and $50 \mathrm{eV}$. The values of the ratio at the plateau, about 0.6 for $\mathrm{D}_{2} \mathrm{O}$ and 0.65 for $\mathrm{H}_{2} \mathrm{O}$, do not depend significantly on the size. 
At larger collision energies, the fragmentation cross section even decreases. This means that $40 \%$ [respectively 35\%] of $\mathrm{D}_{2} \mathrm{O}$ [respectively $\mathrm{H}_{2} \mathrm{O}$ ] molecules that directly encounter $\mathrm{D}_{2} \mathrm{O}$ [respectively $\mathrm{H}_{2} \mathrm{O}$ ] clusters (those inside the geometrical cross section) get out of the collision process without losing a noticeable amount of energy. Actually, the statistical model presented below shows that less than about $1 \mathrm{eV}$ is deposited inside the cluster if no fragmentation occurs.

This kind of fragmentation behavior, characterized by a maximum in the fragmentation probability plotted as a function of collision energy, has been also observed, in a comparable energy range, for the fragmentation of positively charged fullerenes induced by collisions with $\mathrm{Ne}$ and $\mathrm{Ar}$ [9]. However, the observations of ref. [9] are different from ours. First, in $\mathrm{C}_{60}{ }^{+}+\mathrm{Ar}$ collisions, the maximum fragmentation cross section reaches, within experimental accuracy, the geometrical cross section (in the case of $\mathrm{Ne}$, the maximum is at $80 \%$ of the geometrical cross section). It is clearly not the case for $\left(\mathrm{H}_{2} \mathrm{O}\right)_{n} \mathrm{H}^{+}-\mathrm{H}_{2} \mathrm{O}$ collisions, where absolute cross sections are much lower compared to geometrical cross sections. Furthermore our results are more reliable since they can be compared to the results obtained in attachment experiments. Second, the fall in the cross section at high energy in [9] is mainly attributed to the time available for thermal fragmentation, which decreases as the collision energy increases. As shown by our simulation of thermal evaporation presented in Sec. IV C, which takes into account the propagation time after the collision cell and its variation with size and collision energy, this effect cannot explain the fall of the cross section at high energy in our case.

\section{Thermal effect}

Total fragmentation cross sections are not enough to discriminate between the various collision processes described in the introduction. However our experiment gives more information. First, the initial temperature of the clusters can be varied: it is very likely that the temperature dependence of the cross section be different whether the fragmentation is direct or statistical. Second, the fragmentation patterns also contain relevant information: we postpone the results on fragmentation patterns to the next paragraph and concentrate for now on the temperature effects. We first rule out another temperature effect, namely fragmentation induced by absorption of black body background radiation of the experiment chamber at room temperature. This source of fragmentation can be excluded since the lifetime of protonated clusters exposed to a black body radiation spectrum at $300 \mathrm{~K}$, in the size range $n=5-50$, is larger than $10^{-1} \mathrm{~s}$ [24], which exceeds by far the time of flight of the clusters in our experiment, which is in the $100 \mu$ s range.

The collision-induced fragmentation cross sections observed in the collision $\left(\mathrm{H}_{2} \mathrm{O}\right)_{30} \mathrm{H}^{+}+\mathrm{H}_{2} \mathrm{O}$ are shown in figure 4, for two different initial temperatures, namely $25 \mathrm{~K}$ and $100 \mathrm{~K}$. At those temperatures there is no spontaneous thermal evaporation before the clusters enter the collision cell [22]. If there is indeed a statistical redistribution of the collision energy among all internal degrees of freedom followed by thermal evaporation, the fragmentation rate should depend only on the total internal energy after the collision, which is the sum of cluster's initial thermal energy and collision energy. Then, in a thermal evaporation scheme, increasing the initial temperature of the cluster is expected to lower the collision energy that leads to a given fragmentation rate. It is exactly what is observed by comparing the fragmentation rates obtained at two different initial temperatures, which is a first indication that the main fragmentation mechanism is thermal.

\section{Fragmentation patterns}

Fragmentation patterns may be used in order to discriminate between thermal and direct fragmentation: in the case of thermal fragmentation, they can be reproduced with a model based on 
classical evaporation theories, while in the case of direct fragmentation, the intensities of the peaks, at least those close to the parent, are not expected to be reproduced by a statistical model. On the other hand, the interpretation is model dependent. The model itself is made of two pieces: first, a model for the collision process, which gives the distribution of energy deposited in the cluster; second, a model for the evaporation itself. The second one has been the most studied in cluster physics, and is now well established. Two main analytical approaches can be used, which give roughly the same results for our purpose: an Rice-Ramsperger-Kassel model or a Weisskopf model. They assume that the cluster evaporates monomers by sequential unimolecular decay. We used Engelking's derivation of the Weisskopf model $[25,26]$ to describe this decay. The Weisskopf expression of the decay rate of a cluster made of $n$ monomers and bearing the internal energy $E$ is:

$$
W=8 \pi \mu \sigma_{g e o} v_{0}^{\alpha} h^{\alpha-3} \prod_{i=\alpha n-7}^{\alpha(n-1)-4}(\alpha n-1) \frac{(1-D / E)^{\alpha(n-1)-5}}{E^{\alpha-2}}
$$

where $v_{0}$ is the intermolecular vibrational frequency, $\mu$ the reduced mass, $\sigma_{\text {geo }}$ the geometrical cross section as given by Eq. (4), and $\alpha$ the number of degree of freedom per molecule. The sizedependent dissociation energies $D$ are taken from the experimental work by Hansen and coworkers [27].

The internal energy of clusters as a function of their temperature ( $E$ in relation (5)), is given by the Einstein model for the phonon statistics:

$$
E=(\alpha n-6) \frac{h v_{0}}{2}+\frac{(\alpha n-6) h v_{0}}{\exp \left(h v_{0} / k_{B} T\right)-1}
$$

where $k_{B}$ is the Boltzmann constant. In the temperature range (25K-100K) covered in our fragmentation experiments, the internal energy calculated using Eq. (6) is close to that determined for water clusters by Hock and coworkers [28], who measured the heat capacity of negatively charged water clusters for $n=48$ and $n=118$. The heat capacities per molecules of water clusters, which do not vary noticeably between the two sizes 48 and 118, are not expected to vary dramatically from negatively to positively charged clusters [30].

Note that the number of degrees of freedom per molecule $\alpha$ has not been fixed so far to a particular value in the above relations (5) and (6). As a matter of fact, this parameter cannot be determined unambiguously and must be considered in such a simplified model as an effective value. First, this quantity varies with temperature: For instance, the number of degrees of freedom per molecule deduced from the ice caloric curve varies between 0.5 and 4.5 when the temperature goes from $25 \mathrm{~K}$ to $270 \mathrm{~K}$.. Furthermore some internal modes might not couple well with the dissociation coordinates [15]. Moreover, there are discrepancies in the literature concerning the effective number of degrees of freedom of water clusters: Hock et al find that their caloric curve is similar to the one of bulk ice at low temperature [28] whereas others deduce a value close to $6 k_{B}[29,30]$, much higher than the one deduced from bulk ice caloric curve. Anyway, changing the value of the number of degree of freedom in eq. (5) can be easily compensated by slightly changing the prefactor.

We performed several calculations using a number of degrees of freedom varying from 3 to 6 per molecule. The frequency $v_{0}$ needed to be slightly adjusted, but remained always close (within $\pm 20 \%$ ) to the water intermolecular stretch vibrational energy $v_{0}=5.5 \times 10^{12} \mathrm{~Hz}$ [31]. The results presented in figures 1 and 4 were calculated with $\alpha=6, v_{0}=6.5 \times 10^{12} \mathrm{~Hz}$ and $\alpha=3, v_{0}=5.5 \times 10^{12} \mathrm{~Hz}$. Any value between 3 and 6 gives a comparable agreement between experiment and theory. 
Since the energy transfer during the collision depends in a complicated manner on the impact parameter and the initial orientation of the cluster, it is much harder to model the collision process. We used the well-known line of centers model [17], which assumes that the energy transferred $E_{t}$ is the collision energy diminished by the centrifugal energy at distance $R^{*}=R_{c}+R_{0}$, as shown in Fig. 5:

$$
E_{t}=\frac{1}{2} \mu v_{\text {radial }}^{2}=E_{c}\left(1-b^{2} / R *^{2}\right)
$$

When the statistical distribution of impact parameters is taken into account, the distribution of deposited energy is uniform between 0 and the maximum available energy, that is, the collision energy.

Using a Monte-Carlo sampling of the energy transferred, fragmentation patterns and dissociation cross sections can both be simulated using the above models. The comparison with experimental results is shown as solid lines on Fig. 1 for fragmentation patterns and Fig. 4 for cross sections. The agreement is very satisfactory, which indicates that sequential unimolecular evaporation is the most probable fragmentation mechanism in our experiments. However, to reproduce the experimental results in both cases, we had to adjust the only free parameter of the model, namely the molecular radius $R_{0}$ : we had to take a molecular radius $R_{0}=1.62 \AA$, much below the value deduced from either attachment cross sections or ice density $\left(R_{0}=1.98 \AA\right)$. This means that the cross sections are reduced by a factor of about 0.67 respective to the geometric ones. On the other hand, the fragmentation patterns do not depend on the choice of $R_{0}$, since the evaporation rates computed with Eq. (5) are rather insensitive to the prefactors.

The main conclusion of this section on experimental results is that all the observed effects are compatible with a thermal mechanism, except that roughly $35 \%$ of the head-on collision do not lead to fragmentation and thus are unobservable in our experiment.

\section{Discussion}

We first want to stress that the above value of $35 \%$ for non-fragmenting collision is huge: for instance, the experimental fragmentation cross section of $\left(\mathrm{H}_{2} \mathrm{O}\right)_{30} \mathrm{H}^{+}$at a collision energy of $40 \mathrm{eV}$ (with an initial cluster temperature of $25 \mathrm{~K}$ ) is $140 \AA^{2}$, whereas the geometrical cross section is 210 $\AA^{2}$ : this means that the experimental fragmentation probability is $65 \%$, and $35 \%$ of the collisions that occurs within the hard sphere cross section do not undergo fragmentation. On the other hand, the Weisskopf model (Eq. (5)) predicts that the fragmentation time becomes of the order of the observation time when the energy transferred is about $1.5 \mathrm{eV}$. If the hypothesis of uniform distribution of the transferred energy were true, this would mean that at most $4 \%$ of the collisions within the geometrical cross section should be non-fragmenting at $40 \mathrm{eV}$ collision energy.

It is hard to interpret the above result without detailed simulations. In the following, we propose a few hypotheses, which might lead to the observed effect. A first possibility is that the incoming molecule undergoes an elastic collision with a unique molecule of the cluster. However, either the target molecule stays in place, and it receives less than about $1.5 \mathrm{eV}$, which means that the impinging molecule undergoes a very small deviation, and has a high probability to interact with another molecule of the cluster, or the whole energy (minus maybe about $1.5 \mathrm{eV}$ ) is transferred to the target molecule, which is then ejected. We have ruled out this second process by performing collision 
between $\mathrm{H}_{2} \mathrm{O}$ clusters and $\mathrm{D}_{2} \mathrm{O}$ molecules, which show negligible exchange. And the first process is very likely to eventually deposit a significant amount of energy inside the cluster.

Another possibility is that the interaction between the molecule dipole and the cluster charge is repulsive, due to the orientation of the dipole, which for some reason remains frozen during the collision. This could explain why the impinging molecule would be substantially deviated without exciting the cluster, since the repulsion would be at long distance. We have no idea however of the probability of such an event. We can anticipate that it might be dependent on the collision time, since the dipole orientation and the cluster vibration have to be considered frozen.

Actually, a striking feature is displayed in Fig. 6, which might support the hypothesis of a temporal effect related to the vibrational excitation of the clusters. In this figure, the fragmentation cross sections are plotted as a function of the interaction time. The collision time as a function of size and kinetic energy in the lab frame is given by [22]:

$$
2 n^{1 / 3} R_{0}\left(2 E_{k} / n m+3 k_{B} T / m\right)^{-1 / 2}
$$

Here, $n$ is the number of molecules in the cluster, $m$ and $R_{0}=1.98 \AA$ are the mass and radius of a single water molecule, respectively, $T$ is the temperature of the collision cell. Fig. 6 shows that, for collision times greater than the intermolecular vibrational period (deduced from the value of $v_{0}$ used in expressions (5) and (6) ), the energy transfer, thus the fragmentation rate, increases as expected with increasing the collision energy (i.e. with reducing the interaction time). However, when the collision time becomes smaller than the intermolecular vibrational period, the fragmentation rate stops increasing with collision energy, and even decreases for $\mathrm{D}_{2} \mathrm{O}$ clusters. In this case, the energy transfer decreases as the interaction time decreases. In the frame of simple impulsive models where the impinging water molecules hits an individual water molecule of the cluster, this behavior cannot be supported by classical approaches such as the well known "spectator model" [17] in which the energy transfer is expected to be more efficient as the interaction time decreases.

A third hypothesis is that the impinging molecule goes through the cluster without transferring a noticeable energy, as observed in numerical simulations by U. Saalmann and R. Schmidt [3] on sodium clusters. Of course, the structures of sodium and water clusters are different. However, it is known that water can form clathrate structures, with a lot of empty space inside, and it has been shown that water clusters in our size range are amorphous [32]. Whether those structures might allow or hinder an energy-lossless traversal is of course unknown. As pointed out in [3], when the collision time becomes smaller than the typical vibrational period of the cluster, the energy transfer between the projectile and the target becomes less efficient. This is again in agreement with the behavior shown in Fig. 6 .

\section{Conclusion}

Measurements of collision-induced fragmentation cross sections in water cluster-water molecule collisions have shown that the part of collision energy transferred into cluster's internal energy is significantly smaller than expected in the collision energies window 5-100 eV, where $35 \%$ of the collisions that occur within the geometric cross section do not lead to fragmentation although the energy transferred to the cluster is expected to be by far large enough to induce thermal evaporation. 
The measured fragmentation cross sections are compatible with an interpretation in the frame of a thermal evaporation model. It has been shown that, according to the hypothesis of statistical thermal decay, the relevant parameter for calculating the evaporation rate is the total internal energy, that is the sum of the initial thermal energy plus the collision energy, and should consequently depend on both collision energy and initial cluster temperature: At constant collision energy, in this energy range, the measured fragmentation rate indeed varies with the temperature of the cluster, in good agreement with the predictions of the thermal model.

For collision energies ranging from about $5 \mathrm{eV}$ up to tens of $\mathrm{eV}$ however, the fragmentation cross sections of water clusters colliding with single water molecules is roughly constant, and reach a value significantly smaller than the geometric collision cross section. This means that, in this energy range, water molecules hit water clusters at higher and higher velocity without increasing the cluster internal energy, as if water clusters were partially "transparent". At the energies considered, the collision energy is likely to be transferred directly to the cluster's vibrational modes without involving electronic excitation.

This puzzling behavior is not yet really understood. However, it is worth noting that it seems to be similar, to some extent, to the one predicted for sodium-rare gas collisions by U. Saalmann and R. Schmidt [3], who ascribed tentatively this vibronic transparency effect to the relative velocity between projectile and target becoming too large to excite cluster's vibrational degrees of freedom. 


\section{references}

1 S. Díaz-Tendero, L.F. Ruiz, B. Zarour, F. Calvo, F. Spiegelman, P.-A. Hervieux, F. Martín, J. Hanssen, and M.F. Politis, Eur. Phys. J. D 44, 525 (2007)

2 O. Knospe, J. Jellinek, U. Saalmann, R. Schmidt, Phys. Rev. A 61, 022715 (2000)

3 U. Saalmann and R. Schmidt, Phys. Rev. Lett. 80, 3213 (1998)

4 T. Kunert and R. Schmidt, Phys. Rev. Lett. 86, 5258 (2001)

5 C. Bréchignac, Ph. Cahuzac, B. Concina, J. Leygnier, L. F. Ruiz, B. Zarour, P. A. Hervieux, J. Hanssen, M. F. Politis, and F. Martín, Phys. Rev. A 68, 063202 (2003)

6 T. Schlathölter, O. Hadjar, R. Hoekstra, and R. Morgenstern, Phys. Rev. Lett. 82, 73 (1999)

7 S. Tomita, J.S. Forster, P. Hvelplund, A.S. Jensen, abd S.B. Nielsen, Eur. Phys. J. D 16, 119-122 (2001)

8 C. Bréchignac, Ph. Cahuzac, B. Concina, J. Leygnier, L. F. Ruiz, B. Zarour, P. A. Hervieux, J. Hanssen, M. F. Politis, and F. Martín, Phys. Rev. Lett. 89, 183402 (2002)

9 R. Ehlich, M. Westerburg, and E.E.B. Campbell, J. Chem. Phys. 104, 1900 (1996)

10 T. E. Cooper and P. Armentrout, J. Chem. Phys. 134, 114308 (2011)

11 P.E. Barran, N.R. Walker, A.J.Stace, J. Chem. Phys. 112, 6173-6177 (2000)

12 H. Udseth, H. Zmora, R. J. Beuhier, and L. friedman, J. Phys. Chem. 86, 612-617 (1982)

13 K. A. Cowen, B. Plastridge, D. A. Wood, and J. V. Coe, J. Chem. Phys. 99, 3480 (1993)

14 P.H. Dawson, Int. J. Mass Spectrom. Ion Phys., 43, 195 (1982)

15 N. F. Dalleska, K. Honma and P. B. Armentrout, JACS, 115, 12125 (1993)

16 K. Honma and P. B. Armentrout, J. Chem. Phys. 121, 8307-8320 (2004)

17 R. D. Levine and R. B. Bernstein, Molecular reaction dynamics and chemical reactivity, Oxford University Press, New York, 1987

18 L.C. Feldman, J.W. Mayer and S.T.Picraux, Material Analysis by lon Channelling, (1982) Academic Press, New York

19 F. Chirot, S. Zamith, P. Labastie and J.-M. L'Hermite, Rev. Sci. Instrum. 77, 063108 (2006)

20 S. Zamith, P. Feiden, P. Labastie, and J.-M. L'Hermite, J. Chem. Phys. 133, 154305 (2010)

21 F. Chirot, P. Labastie, S. Zamith, and J.-M. L'Hermite, Phys. Rev. Lett. 99, 193401 (2007)

22 S. Zamith, P. Feiden, P. Labastie, and J-M L'Hermite, Phys. Rev. Lett. 104, 103401 (2010)

23 N. C. Lang, H. V. Lilenfeld, J. L. Kinsey, J. Chem. Phys. 55, 3114 (1971)

24 T. Schindler, C. Berg, G. Niedner-Schatteburg, V. E. Bondydey, Chem. Phys. Lett. 250, 301 (1996)

25 V. Weisskopf, Phys. Rev. 52 (1937) 1937

26 P. C. Engelking, J. Chem. Phys. 87 (1987) 936

27 K. Hansen, P. U. Andersson, and E. Uggerud, J. Chem. Phys. 131, 124303 (2009)

28 C. Hock, M. Schmidt, R. Kuhnen, C. Bartels, L. Ma, H. Haberland, and B. v. Issendorff, Phys. Rev. lett. 103, 073401 (2009)

29 Z. Shi, J.V. Ford, S. Wei and A. Castleman, Jr., J. Chem. Phys. 99, 8009 (1993)

30 A. E. Sundén, K. Støchkel, S. Panja, U. Kadhane, P. Hvelplund, S. Brøndsted Nielsen, H. Zettergren, B. Dynefors, and K. Hansen, J. Chem. Phys. 130, 224308 (2009)

31 H. Yada, M. Nagai, K. Tanaka, Chem. Phys. Lett. 473, 279-283 (2009)

32 G. Torchet, J. Farges, M. F. de Feraudy and B. Raoult, Ann. Phys.Fr. 14, 245 (1989) 


\section{Figure captions}

\section{Figure 1:}

Experimental relative intensities of fragments $\left(\mathrm{H}_{2} \mathrm{O}\right)_{n} \mathrm{H}^{+}$(black lines) from the collision of $\left(\mathrm{H}_{2} \mathrm{O}\right)_{30} \mathrm{H}^{+}$ with $\mathrm{H}_{2} \mathrm{O}$ molecules at four center-of-mass collision energies $\mathrm{E}_{\mathrm{cm}}$. Continuous and dashed red lines are obtained from Monte Carlo simulations (Continuous line: $3 n-6$ degrees of freedom, $v_{0}=5.5 \mathrm{THz}$, Dashed line: $6 n-6$ degrees of freedom, $v_{0}=6.5 \mathrm{THz}$, see text).

\section{Figure 2:}

Experimental data points for $-\ln (\mathrm{I} / \mathrm{lo}$ ) (see relation (1)) as a function of the product of water vapor density in the cell times the cell length. The continuous line is a linear fit through the data points. The fit yields a value of $125 \AA^{2}$ for the fragmentation cross-section. The data points are obtained for $\left(\mathrm{H}_{2} \mathrm{O}\right)_{30} \mathrm{H}^{+}$clusters at a collision energy of $2.3 \mathrm{eV}$.

\section{Figure 3:}

Fragmentation cross section, relative to the geometrical cross section, plotted for various cluster sizes and collision energies. a) Fragmentation of deuterated heavy water clusters induced by collisions with heavy water molecules. b) Fragmentation of protonated water clusters induced by collisions with water molecules.

\section{Figure 4:}

Cross section of the fragmentation of $\left(\mathrm{H}_{2} \mathrm{O}\right)_{30} \mathrm{H}^{+}$induced by collisions with $\mathrm{H}_{2} \mathrm{O}$ molecules as a function of collision energy for two different initial temperatures of the cluster ( $25 \mathrm{~K}$ and $100 \mathrm{~K}$ ). The continuous and dashed lines are obtained from a Monte Carlo simulation using the model described in the text. Continuous line: $3 n-6$ degrees of freedom, $v_{0}=5.5 \mathrm{THz}$, Dashed line: $6 n-6$ degrees of freedom, $v_{0}=6.5 \mathrm{THz}$. The straight line (red online) at $210 \AA^{2}$ is the geometrical cross section.

\section{Figure 5:}

The radial energy transfer: only the energy transferred along the radial component $v_{\text {radial }}$ is assumed to be transferred into internal energy.

\section{Figure 6:}

Fragmentation cross section, relative to the geometrical cross section, plotted as a function of the duration of the collisions for different cluster sizes for $\mathrm{D}_{2} \mathrm{O}\left(\right.$ a) and $\mathrm{H}_{2} \mathrm{O}$ (b) clusters. The vertical lines indicate the vibrational period deduced here from $v_{0}=5.5 \mathrm{THz}$. 


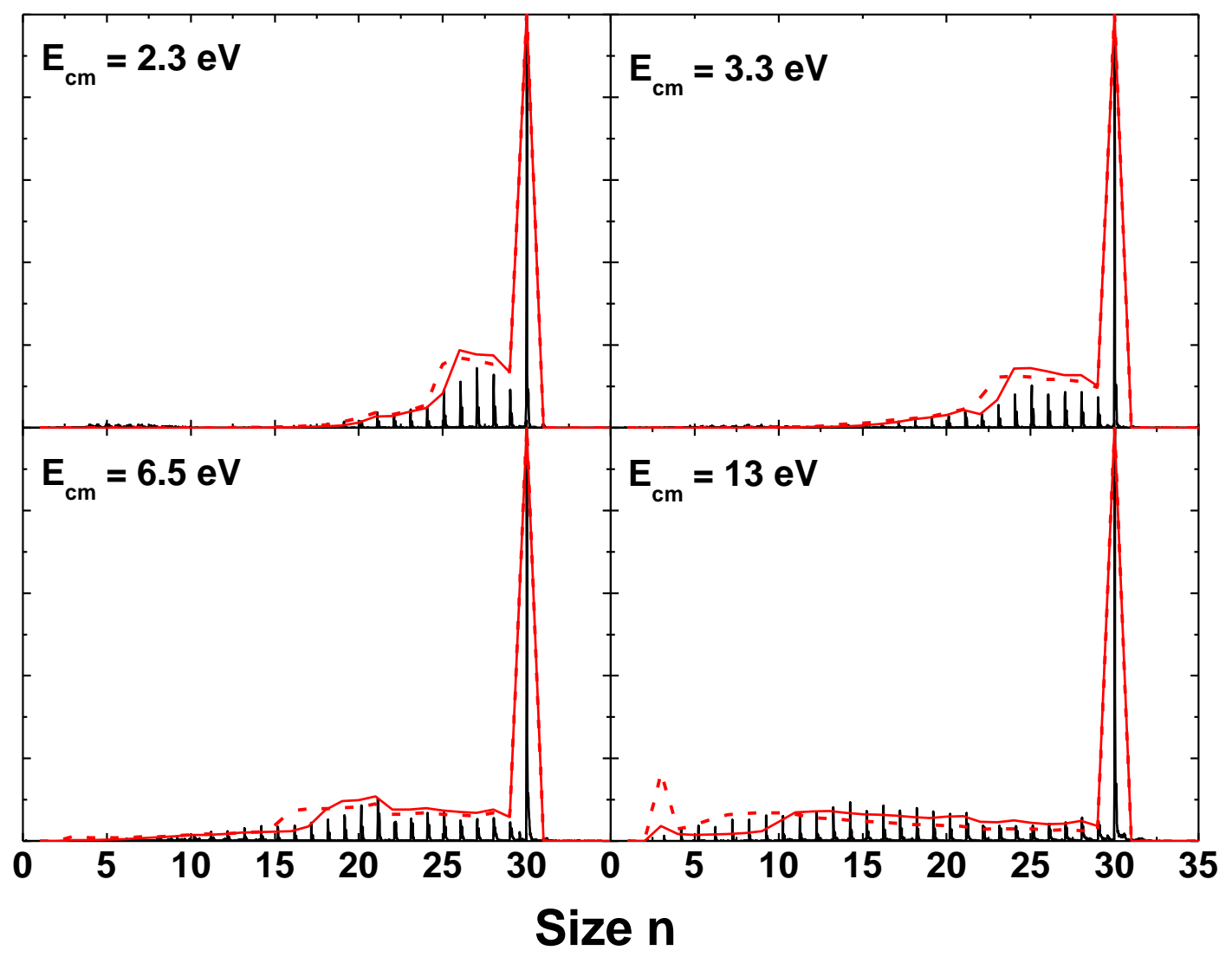

Figure 1 


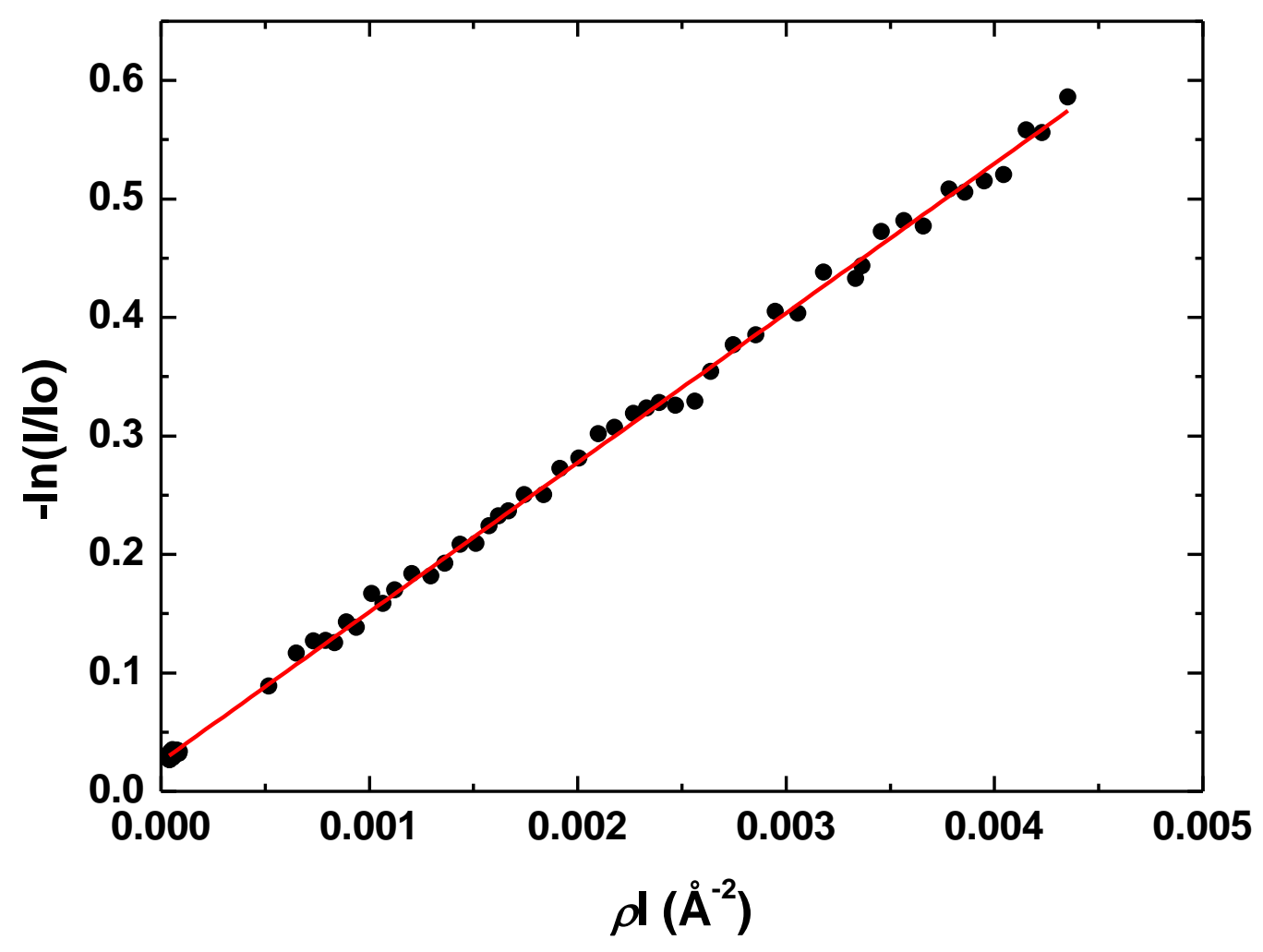

Figure 2 


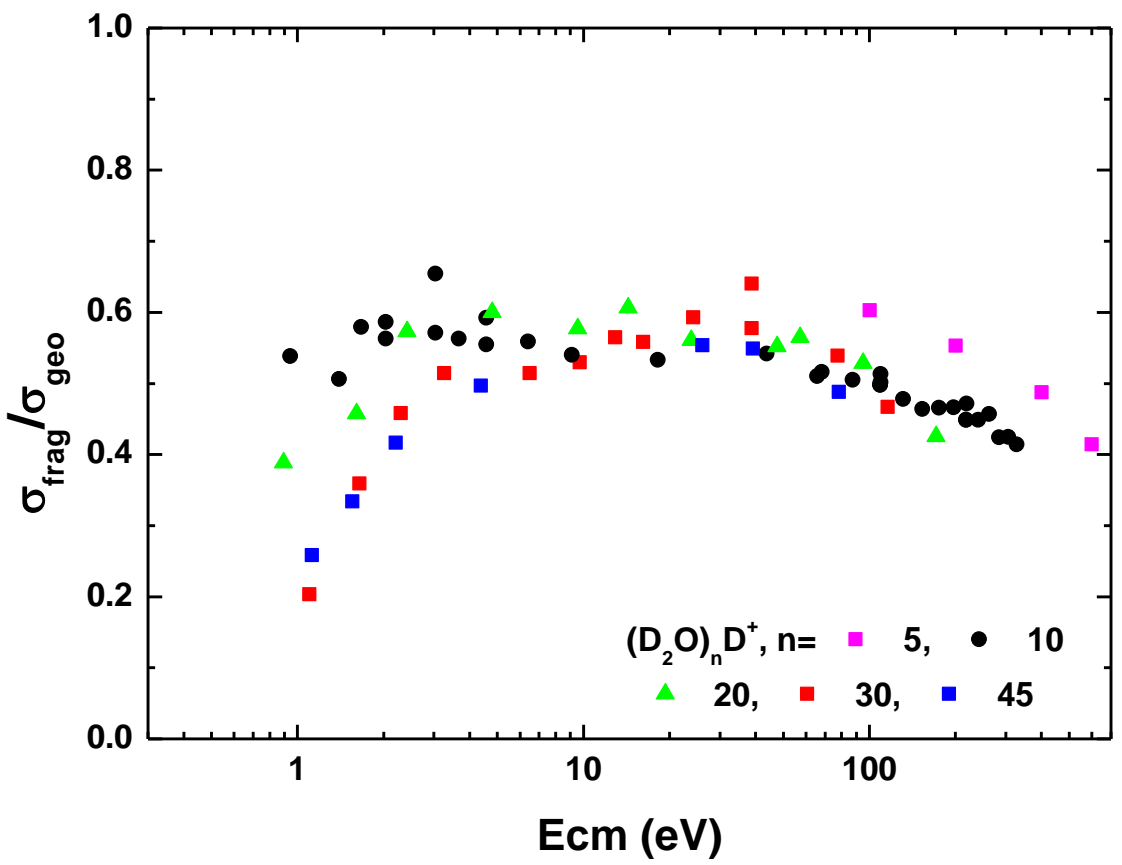

Figure 3 a)

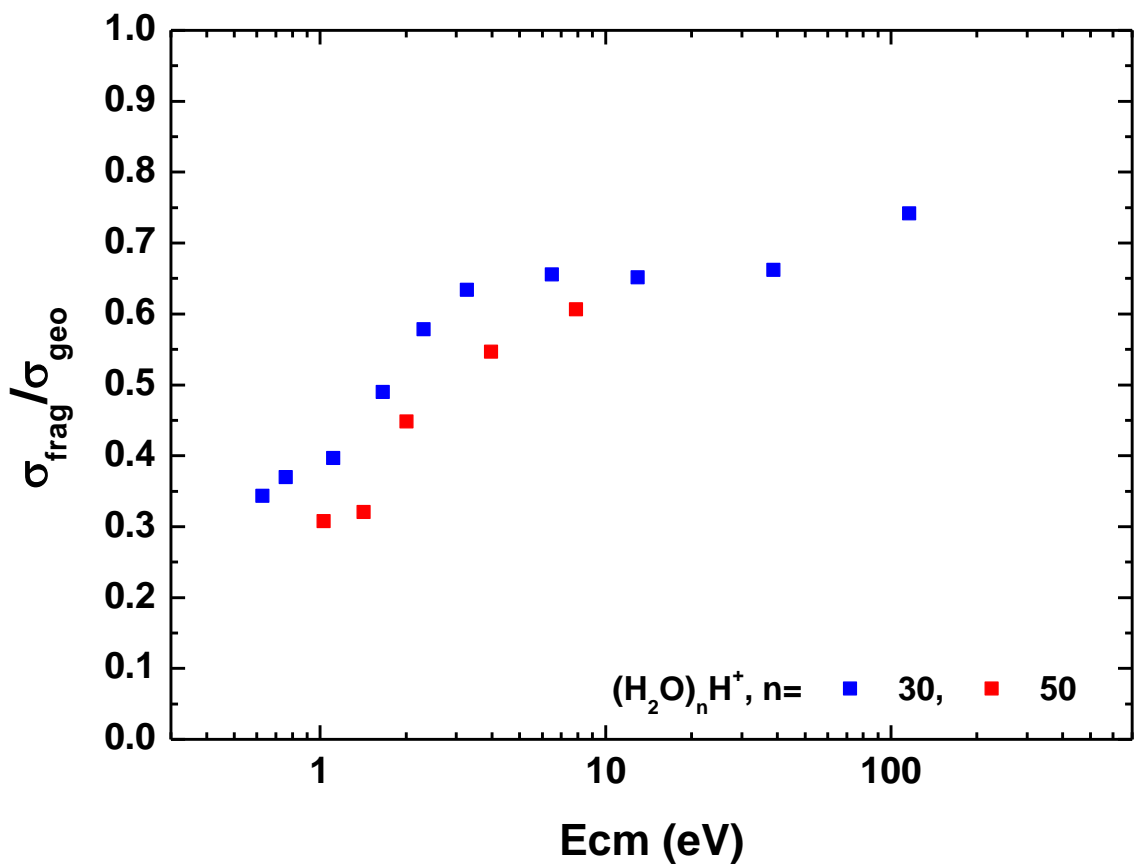

Figure 3 b) 


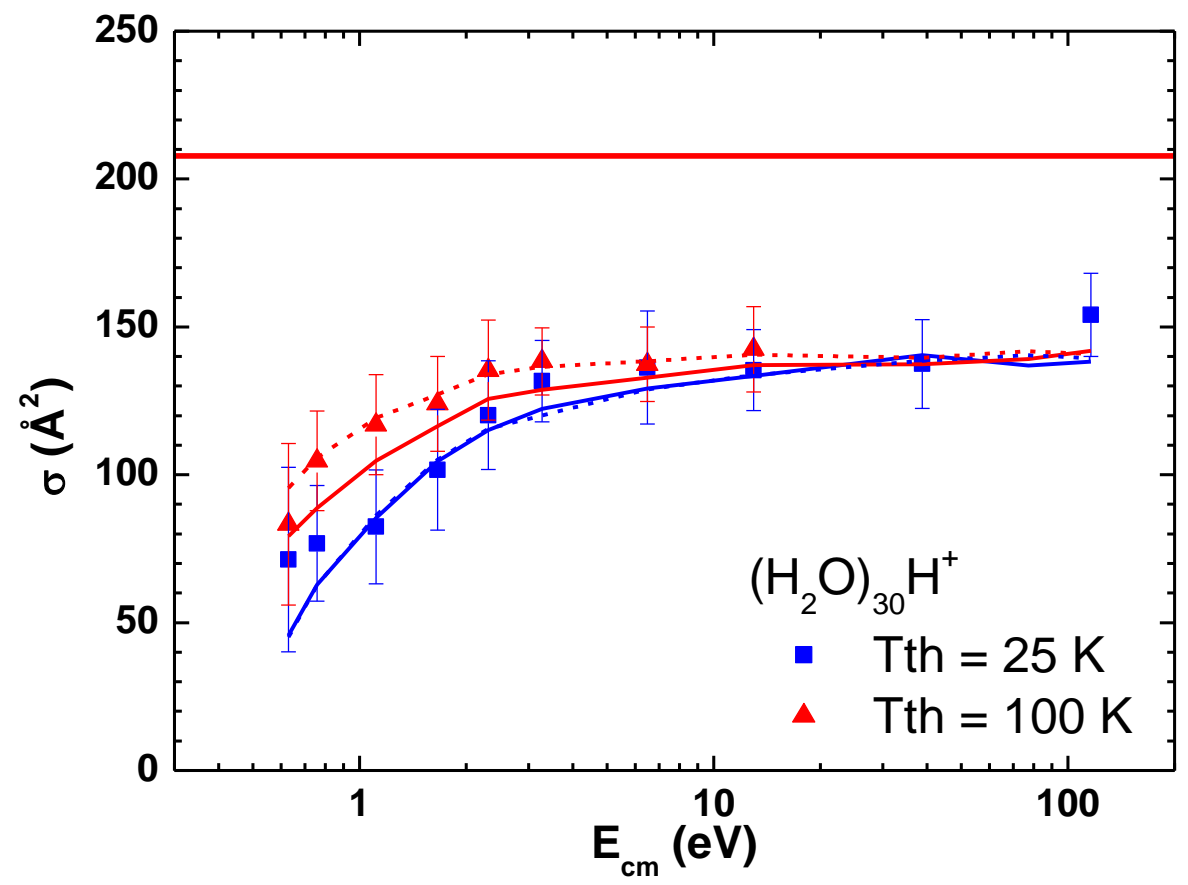

Figure 4 


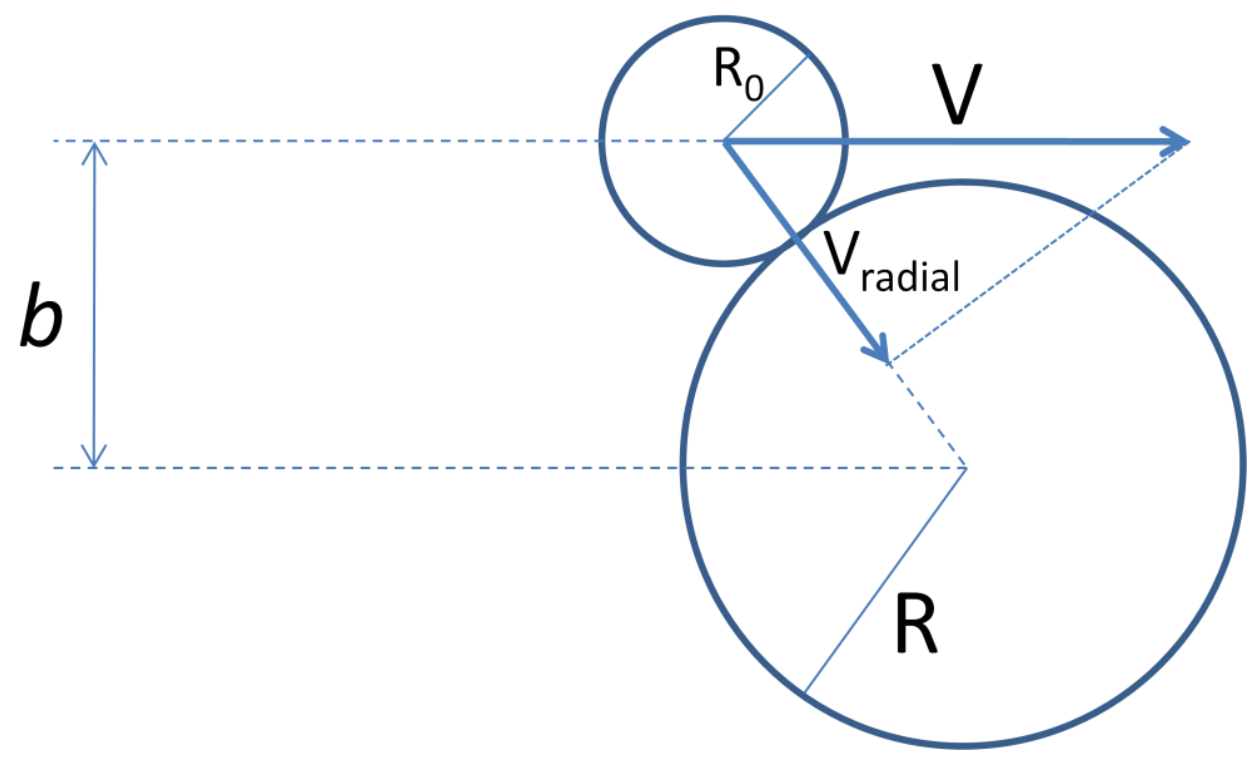

Figure 5 


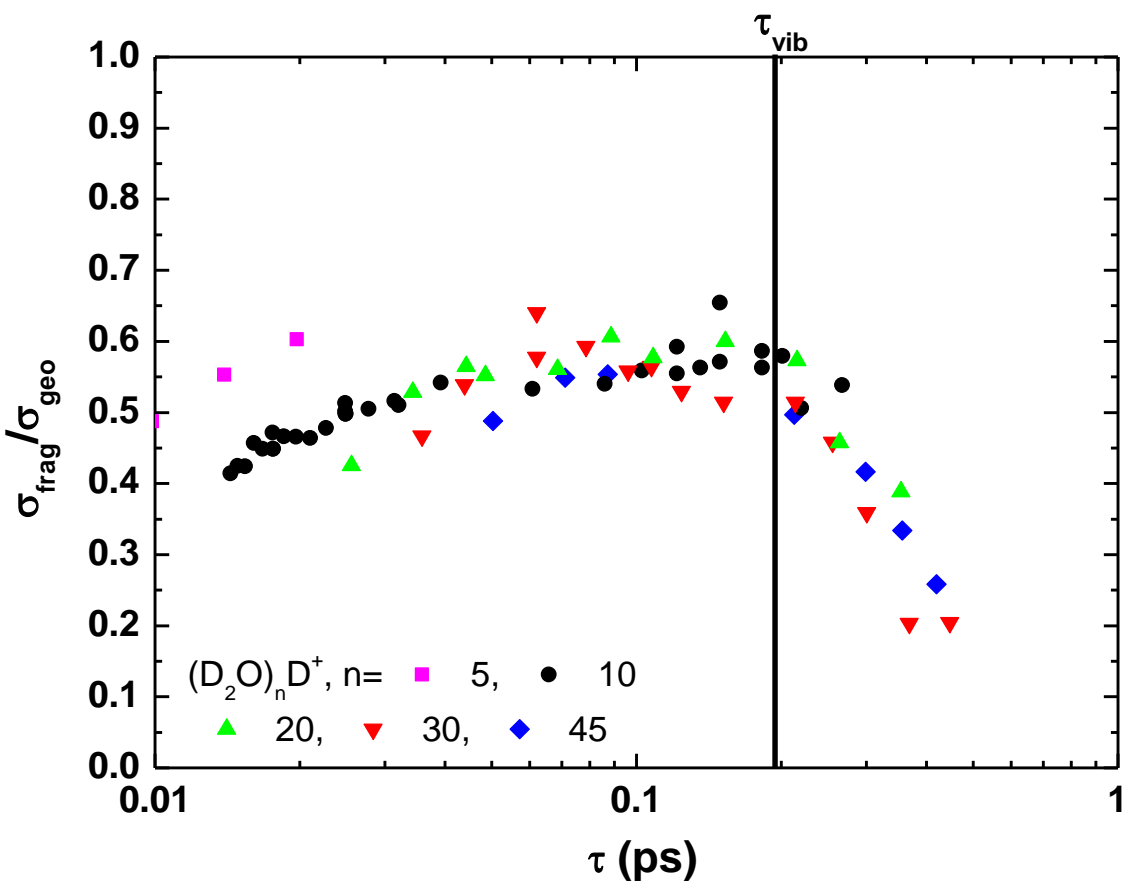

Figure 6 a)

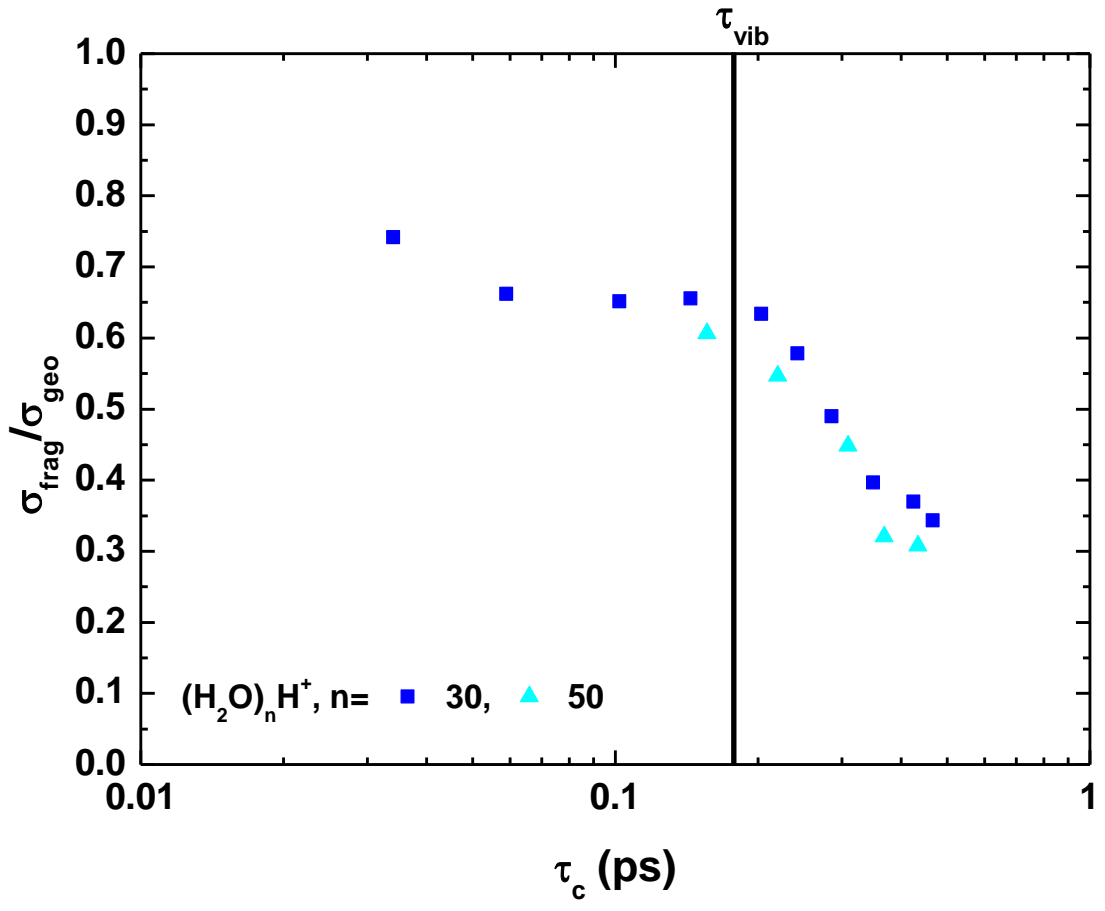

Figure 6 b) 